\title{
Minimal Webs in Riemannian Manifolds
}

\section{Markvorsen, Steen}

\section{Published in:}

Geometriae Dedicata

Link to article, DOI:

10.1007/s10711-008-9230-8

Publication date:

2008

\section{Document Version}

Early version, also known as pre-print

Link back to DTU Orbit

Citation (APA):

Markvorsen, S. (2008). Minimal Webs in Riemannian Manifolds. Geometriae Dedicata, 133(1), 7-34. https://doi.org/10.1007/s10711-008-9230-8

\section{General rights}

Copyright and moral rights for the publications made accessible in the public portal are retained by the authors and/or other copyright owners and it is a condition of accessing publications that users recognise and abide by the legal requirements associated with these rights.

- Users may download and print one copy of any publication from the public portal for the purpose of private study or research.

- You may not further distribute the material or use it for any profit-making activity or commercial gain

- You may freely distribute the URL identifying the publication in the public portal

If you believe that this document breaches copyright please contact us providing details, and we will remove access to the work immediately and investigate your claim. 


\title{
MINIMAL WEBS IN RIEMANNIAN MANIFOLDS
}

\author{
STEEN MARKVORSEN
}

\begin{abstract}
For a given combinatorial graph $G$ a geometrization $(G, g)$ of the graph is obtained by considering each edge of the graph as a 1-dimensional manifold with an associated metric $g$. In this paper we are concerned with minimal isometric immersions of geometrized graphs $(G, g)$ into Riemannian manifolds $\left(N^{n}, h\right)$. Such immersions we call minimal webs. They admit a natural 'geometric' extension of the intrinsic combinatorial discrete Laplacian. The geometric Laplacian on minimal webs enjoys standard properties such as the maximum principle and the divergence theorems, which are of instrumental importance for the applications. We apply these properties to show that minimal webs in ambient Riemannian spaces share several analytic and geometric properties with their smooth (minimal submanifold) counterparts in such spaces. In particular we use appropriate versions of the divergence theorems together with the comparison techniques for distance functions in Riemannian geometry and obtain bounds for the first Dirichlet eigenvalues, the exit times and the capacities as well as isoperimetric type inequalities for so-called extrinsic $R$-webs of minimal webs in ambient Riemannian manifolds with bounded curvature.
\end{abstract}

\section{INTRODUCTION}

We let $G=(V, E)$ denote an abstract infinite graph with edge set $E$ and vertex set $V$. We will use standard notation and terminology from graph theory, see e.g [We], [MT], [Wo]. For example, two vertices $x$ and $y$ in $V$ are called neighbours if there is at least one edge $e$ in $E$ between them, in which case we write $x \sim y$ and $e=x y$. Multigraphs (with a finite number of multiple edges between neighbouring vertices) are allowed. Loops (pseudo-graphs) are not allowed. In other words we assume without lack of generality that graphs containing loops have been 'normalized' by introducing an auxiliary vertex somewhere on every loop edge. We also assume that the graph is countable and connected as well as locally finite (but not too finite) in the sense that every vertex $p \in V$ has finite vertex degree $m(p) \geq 2$.

2000 Mathematics Subject Classification. Primary 53C, 05C, 58J, 60J.

Key words and phrases. Minimal immersions, locally finite countable graphs, extrinsic minimal $R$-webs, Laplacian, eigenvalues, capacity, transience, isoperimetric inequalities, comparison theory. 
We geometrize the graph $G$ as follows. Every edge $e=x y$ in $E$ is considered as a compact 1-dimensional manifold with boundary $\partial e=x \cup y$ (where $x$ and $y$ are the vertices in $E$ which are joined in $G$ by $e$ ). Let each edge $e$ be given a metric $g_{e}$ such that $\left(e, g_{e}\right)$ is isometric to a finite interval $[0, L(e)]$ of the real line with the standard metric. We assume throughout that $L(e)>\varepsilon$ for some positive $\varepsilon$ for every edge $e \in E$. The distance metric on the edges can be extended to the full graph via infima of lengths of curves in the geometrization of $G$. Then the graphs become metrically complete length spaces, see e.g. [BH, Chapter 1.3]. In particular, for every two points $p, q$ in the geometric graph there exists a minimal geodesic joining $p$ and $q$. The distance $d(p, q)$ in $\mathrm{G}$ between $p$ and $q$ is the length of such a geodesic. Note that because of the assumption $m(p) \geq 2$ every geodesic can be extended in such a way that the extension is still the shortest connection between any pair of points - at least locally. However, the extension through a vertex point may not be unique.

The resulting length space is called $(G, g)$ - or just shorthand $G$. We note that the intrinsic curvature at every vertex with degree $m \geq 3$ is $-\infty$ in the geodesic triangle comparison sense, see e.g. [BuBI]. In such cases $(G, g)$ does not have bounded geometry in this geometric sense, but only in the combinatorial sense of having bounded degree.

1.1. Concerning the literature on metric graphs and webs. The intrinsic discrete analysis of functions defined only on the vertices of a given graph has produced a wealth of results beginning with the works of $[\mathrm{E}],[\mathrm{H}]$, and [Dod2]. We find excellent surveys in e.g. [Ch], [So], $[\mathrm{Wo}]$, and $[\mathrm{CoG}]$.

The idea of extending the analysis to intrinsic differentiable functions defined on the full edges of the graph has been considered from different viewpoints. We refer to J. Friedman $[\mathrm{Fr}]$ and Y. Ohno and $\mathrm{H}$. Urakawa [OU], who obtain results concerning the eigenvalues of the discrete Laplacian on graphs by way of linear interpolation along the edges.

When we impose natural (Kirchhoff type) conditions at the vertices, the spectrum of the discrete Laplace operator and the corresponding eigenfunctions (defined at the vertices) determine the spectrum of the geometric Laplacian and the continuous (Kirchhoff) eigenfunctions. This far reaching intrinsic relationship has been studied by a number of authors, see e.g. [Ro], [Nic], [Be], [Cat], [FT], and the excellent recent survey papers on quantum graphs: [EP, Ku1, Ku2, SmSo]. 
Yet another main idea of the present paper is to facilitate the analysis of, say, eigenvalues and isoperimetric properties of metric graphs by appealing to the fruitful interplay between the 'inner' combinatorial geometry and the 'outer' geometry of graphs which are immersed isometrically and minimally into a given ambient Riemannian manifold. A related point of view has been applied in $[\mathrm{ChY}]$, where F. Chung and S. T. Yau obtain lower bounds on Neumann eigenvalues of certain subgraphs of homogeneous lattice graphs embedded into Riemannian manifolds. In their setting the eigenvalue bounds are derived from known results for eigenvalues of the ambient Riemannian manifolds using both the discrete heat kernels of the graphs and the continuous heat kernels of the Riemannian manifolds in question.

In the other direction we refer to the work of K. Fujiwara in [Fu], where he obtains a two-sided estimate of the spectrum of a given compact Riemannian manifold via the discrete spectra of roughly isometric nets on the manifold in the sense of Kanai, see [Ka1, Ka2, Ka3]. These nets, however, do not necessarily span minimal webs in the sense of Definition 2.6 below. It is an open question - stated in [Fu, p. 2587] - whether a suitable 'nice' sequence of graphs could improve the estimation of the eigenvalues of compact manifolds. At this note and in a related vein, H. Urakawa obtains in [U2] explicit limit expressions for the Dirichlet and Neumann eigenvalues from special (equilateral or isosceles right) triangulations of bounded plane domains. In particular the resulting approximating graphs are thence in fact planar minimal webs. The result supports the general idea that minimal webs could be of instrumental value for the precise estimation of the spectra of Riemannian manifolds in general.

Here we also emphasize in particular those aspects of the previous works which are related to the notion of harmonic maps of graphs into suitable target spaces. In [U1], $[\mathrm{A}]$, and [Ts] the harmonic morphisms of (weighted) graphs into (weighted) graphs is defined and studied. As observed by C. K. Anand in [A], Remark 3, the discrete combinatorial structure of the target spaces in such a setting does not, however, allow directly for a proper definition of an energy functional (whose critical maps should then be called harmonic). The work [EF] by Eells and Fuglede offers a natural setting for the study of harmonic maps of general Riemannian polyhedra into Alexandrov spaces.

In the present paper we consider only those maps on metric graphs which are isometric immersions and minimal in a sense, which will be made precise in the next section. 
Acknowledgement The author would like to thank the referee for excellent questions, corrections, and comments, which greatly improved the presentation of this work.

\section{Preliminaries \\ AND OUTLINE OF MAIN RESULTS}

Definition 2.1. A continuous map $\phi$ of $(G, g)$ into a given Riemannian manifold $\left(N^{n}, h\right)$ will be called an isometry if it is an isometric immersion in the usual sense on every edge with respect to the metric induced from $h$.

Remark 2.2. By continuity the isometries of geometrized graphs preserve the global graph structure in the sense that $\phi(e)=\phi(x) \phi(y)$ whenever $e=x y$, whereas they only preserve the local distances of the corresponding metric space continua. In comparison, the isometric embeddings of finite combinatorial metric graphs considered in e.g. [DL] preserve all the distances represented by the full distance matrices of the corresponding finite metric spaces. In both contexts the geometry and topology of the target spaces represent interesting possible obstructions for isometric immersions of a given $(G, g)$ to exist.

Definition 2.3. A given isometric immersion $\phi(G)$ is edge-minimal if the image of each edge is a geodesic segment in $N^{n}$ (realizing locally the distances between pairs of points on each edge).

In the following we shall often use the notation $G$ as shorthand for both $(G, g)$ and $\phi(G, g)$ unless the context calls for special attention concerning the metric $g$ or concerning specific properties of a given isometric immersion $\phi$. In particular we note, that a given edge-minimal isometric immersion of $G$ into $N^{n}$ may map several edges of $G$ into identical (or overlapping segments of) geodesics in $N^{n}$. For example, we may consider immersions of non-line graphs into $\mathbb{R}^{1}$. In such cases it is important to keep track of the combinatorics of the original abstract graph, so that the edges in the immersed image is counted with the correct multiplicities.

To facilitate the local analysis of functions in a given metric neighborhood of a vertex $p$ in $G$ we introduce the notion of parametrized star spaces as follows.

Definition 2.4. The $p$-centered parametrized star space $Y_{p} \subset(G, g)$ is the compact metric subspace of $(G, g)$ consisting of the vertex $p$ together with the arc length parametrized edges emanating from $p$ :

$$
e_{i}=\gamma_{i}\left(\left[0, L\left(e_{i}\right)\right]\right), i=1, \ldots, m(p)
$$

where $\gamma_{i}(s), s \in\left[0, L\left(e_{i}\right)\right]$, denotes the unique point on the edge $e_{i}$ which is at distance $s$ from $p$. 


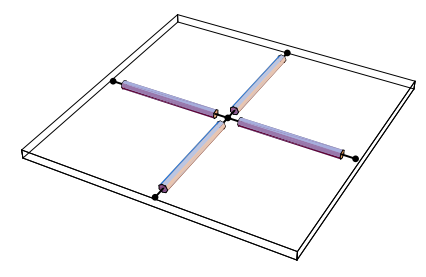

FiguRE 1. A 2D joint element for Scherk's web in $\mathbb{R}^{3}$.

Figures 1 and 2 show special well known star spaces. They will be used to construct the so-called Scherk web in Example 2.7. The Scherk web was originally introduced in $[\mathrm{MMT}]$ as a discrete approximation to Scherk's doubly periodic minimal surface, see Figures 3, 4, and 5.

Definition 2.5. The immersion $\phi(G)$ is vertex-minimal if every vertex is 'edge-balanced' in the following way: Let $p$ denote a given vertex in the image of $G$ in $N^{n}$. Then $\phi(G)$ is vertex minimal at $p$ if the unit tangent vectors to the emanating edges from $p$ in $Y_{p}$ add up to the zero vector in $T_{p} N$, i.e.

$$
\sum_{i=1}^{m(p)} \dot{\gamma}_{i}(0)=0
$$

Definition 2.6. We will say that the immersion $\phi(G)$ is minimal if it is both vertex-minimal and edge-minimal.

An example of a minimal web in $\mathbb{R}^{3}$ which has already been alluded to above is the 'skeleton' of Scherk's surface:

Example 2.7. Scherk's surface is the doubly periodic minimal surface in $\mathbb{R}^{3}$ defined by the Monge patch parametrization $\phi(u, v)=\ln \left(\frac{\cos (v)}{\cos (u)}\right)$. The domain of definition in the $(u, v)$-plane is like the black squares in an infinite checkerboard pattern. In Figure 3 we show one piece of the surface which is defined over just one square - 7 such pieces fit together smoothly, as shown in Figure 5. Every vertex in Scherk's web is the center of a star-space which is one of two types, a $2 \mathrm{D}$ joint as in Figure 1 or a 3D joint as in Figure 2. The Scherk web construction is also shown in Figures 4 and 5. Every vertex is clearly minimal and since every edge is a straight line segment, we conclude: Scherk's web is minimal in $\mathbb{R}^{3}$.

Definition 2.8. We recall that a map $\psi$ between metric spaces $\left(X, d_{X}\right)$ and $\left(Y, d_{Y}\right)$ is called $a$ rough isometry if there are constants $\alpha \geq 1$ and $\beta \geq 0$ such that for every $(x, y) \in X \times X$ we have

$$
\alpha^{-1} d_{X}(x, y)-\beta \leq d_{Y}(\psi(x), \psi(y)) \leq \alpha d_{X}(x, y)+\beta .
$$

Scherk's web is known to be roughly isometric to the doubly periodic Scherk minimal surface in $\mathbb{R}^{3}$; See $[\mathrm{MMT}],[\mathrm{C} 2]$. 


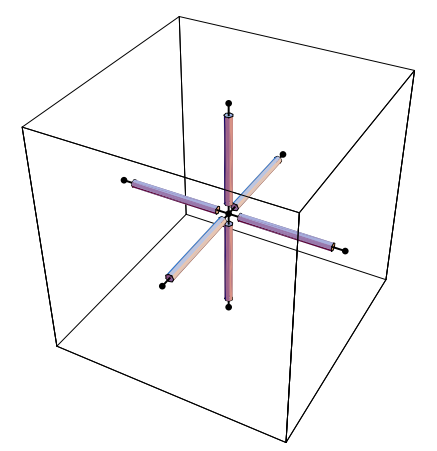

FiguRE 2. A 3D joint element for Scherk's web in $\mathbb{R}^{3}$.

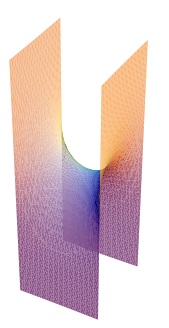

FiguRE 3. A block of Scherk's surface.

Definition 2.9. A given Riemannian manifold $M$ has bounded geometry if the injectivity radius of $M$ is bounded positively away from 0 and if the Ricci curvatures of $M$ are bounded away from $-\infty$.

In view of the examples considered above and in view of the flexibility of the constructions involved, we conjecture that every minimal submanifold in an ambient Riemannian manifold $N$ may be approximated by a roughly isometric, Hausdorff close, minimal web in $N$. The Hausdorff distance between two subsets $A$ and $B$ of $N$ is defined to be the infimum of all $\eta$ for which $A$ is contained in the metric $\eta$-tube around $B$, and $B$ is contained in the metric $\eta$-tube around $A$ in $N$ (see e.g. section 7.3 in $[\mathrm{BuBI}])$.

Conjecture 2.10. Let $P^{m}$ denote the image of a minimally immersed submanifold in a Riemannian manifold $N^{n}$. Suppose that $P^{m}$ and $N^{n}$ have bounded geometries. Let $\varepsilon$ be any given positive number. Then there exists a metric graph $(G, g)$ and a minimal isometric immersion of $(G, g)$ into $N^{n}$, such that $(G, g)$ is roughly isometric to $P^{m}$ and such that the Hausdorff distance $d_{H}\left(G, P^{m}\right)$ between the image of $G$ and $P^{m}$ in $N^{n}$ is less than $\varepsilon$.

A very interesting recent development related to this conjecture is found in the works of Bobenko, Hoffman, Pinkall, and Springborn, see 


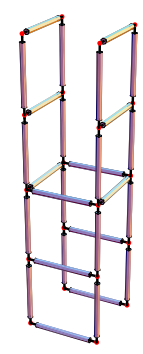

Figure 4. A graph building block for Scherk's web.

e.g. [BP, BHS]. For example they obtain $O\left(\frac{1}{n}\right)$-approximations to minimal surfaces in $\mathbb{R}^{3}$ by constructing discrete Weierstrass representations from discrete holomorphic maps from $\frac{1}{n} \mathbb{Z}^{2}$.

2.1. Main results. Having constructed and analyzed the geometric Laplacian $\Delta^{G}$ on graphs and after having proved the fundamental properties alluded to in the abstract, we show in Section 9 that the first Dirichlet eigenvalue of certain subsets of minimal webs (called $R$-webs, defined in Section 7) are bounded from below by $\pi^{2} / 4 R^{2}$ when the ambient space has an upper curvature bound; See Theorem 9.4 .

A phenomenon, which in fact is related to this eigenvalue estimate is the following: If you get lost in a minimal maze (with the architecture of a minimal $R$-web in $\mathbb{R}^{n}$ ) then you can get out fast by performing a $\Delta^{G}$-driven Brownian motion in the maze. If the ambient space is negatively curved, then you get out even faster unless the web architecture is that of a star web, whose inner geometry is clearly not able to 'feel' the curvature of the ambient space. These results are stated and proved via the notion of mean exit time functions in Theorem 9.6 and in Theorem 8.4; See also Remark 8.5.

In Section 10 we obtain isoperimetric inequalities which show that $R$-web subsets of minimal webs behave much the same way as totally geodesic $R$-discs in space forms: In negatively curved ambient spaces the boundary is large relative to the interior mass and in positively curved ambient spaces the opposite holds true; See Theorem 10.4.

Finally, in Section 11 we show bounds on the capacity of annular subsets of minimal metric webs - see Theorem 11.4 - and we relate these bounds to the notions of transience and recurrence for complete 

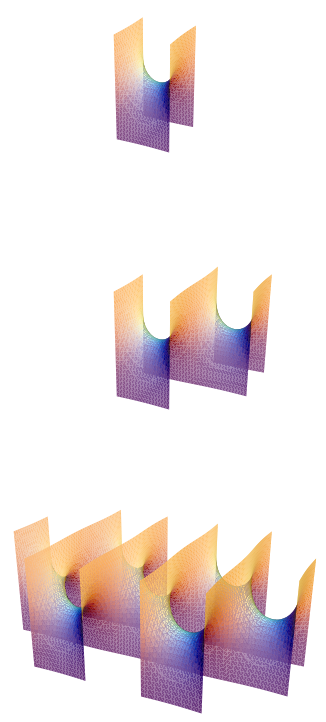
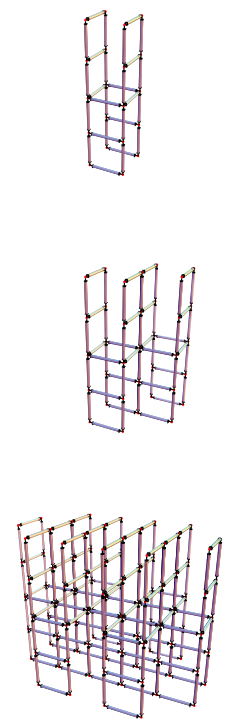

FIGURE 5. Scherk's minimal buildings - surface and web, respectively.

minimal webs in Hadamard-Cartan manifolds; See Corollary 11.7. In terms of the maze analogy alluded to above, an infinite geometric maze is transient if the Brownian motion in it is not certain to visit every vertex as time goes by. In consequence there is a positive probability of getting lost at infinity. We have shown in [MMT] that Scherk's maze is transient. In view of Theorem 8.4 this shows in particular, that the mean exit time functions for $R$-webs are not able to tell if a given infinite minimal web is transient or not.

\section{The GeOMETRIC LAPlacian OF ADMISSIBLE (KIRCHHOFF) FUNCTIONS}

In this section we consider the local analysis (to second order) of functions defined on the geometrized graphs (resp. on their isometric immersions into Riemannian manifolds). In this paper we mainly consider finite precompact subgraphs of the geometrized graphs, in particular the so-called $R$-webs which will be defined shortly in section 7 below. In the following analysis we therefore assume that $G$ is finite. The intrinsic analysis of functions on the open edges of a finite graph is standard and as elementary as can be. Hence we must pay special attention to the notion of second order derivative, i.e. the Laplacian, at the vertices of the finite graphs.

We consider the set $C^{0}(G)$ of continuous functions on $G$. Then $L^{2}(G)=\bigoplus_{j=1}^{\# E} L^{2}\left(e_{j}\right)$, and with $f_{j}=f_{\left.\right|_{e_{j}}}$ we set 


$$
\|f\|_{G}^{2}=\sum_{j}\left\|f_{j}\right\|_{e_{j}}^{2}=\sum_{j} \int_{e_{j}}\left|f_{j}(t)\right|^{2} d t
$$

We let $\mathcal{H}^{1}(G)$ denote the Sobolev space obtained as the completion of the set

$$
\left\{f \in C^{0}(G) \mid f_{j} \in C^{1}\left(e_{j}\right)\right\},
$$

where the closure is with respect to the norm

$$
\|f\|_{1, G}^{2}=\sum_{j}\left(\left\|f_{j}\right\|_{e_{j}}^{2}+\left\|f_{j}^{\prime}\right\|_{e_{j}}^{2}\right)
$$

For all $f \in \mathcal{H}^{1}(G)$ we associate a quadratic form to $G$ :

$$
\mathcal{F}: f \rightarrow\left\|f^{\prime}\right\|_{G}^{2}=\sum_{j}\left\|f_{j}^{\prime}\right\|_{e_{j}}^{2}
$$

The Laplacian of $G$ is then the unique self-adjoint and non-negative operator $\Delta^{G}$ associated with the closed form $\mathcal{F}$, see e.g. [Cat, Lions, EP, D, RS].

Lemma 3.1 (See e.g. [Cat], Lemma 1). The domain $\mathcal{D}_{G}$ of $\Delta^{G}$ consists of all functions $f \in C^{1}(G)$ which are twice weakly differentiable on each edge and which satisfies the Kirchhoff condition at each vertex. The functions in $\mathcal{D}_{G}$ will be called the admissible functions on $G$.

We now explain this latter Kirchhoff condition in some detail because it mimics precisely the geometric condition of vertex minimality previously introduces in 2.5. For each edge $e=\gamma([0, L(e)])$ in the parametrized star space $Y_{p}$ from a point $p$ we have for every $f \in \mathcal{D}_{G}$ :

$$
\left\{\begin{array}{l}
\lim _{s \rightarrow 0} f(\gamma(s))=f(\gamma(0))=f(p) \\
\lim _{s \rightarrow 0} f^{\prime}(\gamma(s))=f^{\prime}(\gamma(0)) \quad \text { and } \\
\lim _{s \rightarrow 0} f^{\prime \prime}(\gamma(s))=f^{\prime \prime}(\gamma(0))
\end{array}\right.
$$

where we use shorthand notation $f^{\prime}\left(\gamma\left(s_{0}\right)\right)$ for the first derivative of the function $f(\gamma(s))$ with respect to $s$ at $s=s_{0}$.

Every function $\psi$ in $\mathcal{D}_{G}$ satisfies

Definition 3.2 (The Kirchhoff condition).

$$
\sum_{i=1}^{m(p)} \psi^{\prime}\left(\gamma_{i}(0)\right)=0 \quad \text { at every vertex } p \text { in } V, \gamma_{i} \in Y_{p}
$$

Remark 3.3. The Kirchhoff condition is a first order 'balancing' condition for the functions at each vertex of the graph $G$. As we shall see in the next section, this property is naturally inherited (by functions which are restrictions to $G$ from $N^{n}$ ) when $G$ is vertex-minimal in $N^{n}$. 
In the domain $\mathcal{D}_{G}$ the Laplacian is related to the form $\mathcal{F}$ as follows:

$$
\left\langle\Delta^{G} f, f\right\rangle_{L^{2}(G)}=\left\|f^{\prime}\right\|_{G}^{2}
$$

The name 'Laplacian' is further motivated by:

Corollary 3.4. Let $f \in \mathcal{D}_{G}$. Along the interior of any given edge $e=\gamma([0, L(e)])$ the Laplacian of $f$ is the usual second order derivative with respect to arclength (independent of the orientation of the parametrization of the edge):

$$
\left.\Delta^{G}(f)_{\left.\right|_{\gamma(s)}}=\frac{d^{2}}{d s^{2}} f(\gamma(s))=f^{\prime \prime}(\gamma(s)) \quad \text { for } s \in\right] 0, L(e)[.
$$

At any given vertex $p$ in $G$ we have - using the parametrized star space $Y_{p}$ :

$$
\Delta^{G}(f)_{\left.\right|_{p}}=\lim _{s \rightarrow 0}\left(\frac{2}{s^{2}}\left[\frac{1}{m(p)} \sum_{i=1}^{m(p)}\left(f\left(\gamma_{i}(s)\right)-f\left(\gamma_{i}(0)\right)\right)\right]\right) .
$$

Remark 3.5. The square bracket in this definition is (when $L(e)=$ 1 for all the edges and $s=1$ ) the discrete combinatorial Laplacian which was studied and applied by Dodziuk and co-workers in [Dod2], [DodKa], [DodKe]. This definition may be considered a natural limit of the combinatorial Laplacian obtained as follows: Subdivide every edge by inserting $w=L(e) / s$ new auxiliary vertices equidistributed along each edge $e$, scale the graph by a homothety with factor $1 / s$, so that the new graph has all its edge-lengths equal to 1 , calculate the usual discrete combinatorial Laplacian of $f$, and finally multiply the result by $2 / s^{2}$. It should come as no surprise, therefore, that this definition of the Laplacian satisfies the important maximum principle. For completeness we give the proof below, cf. Proposition 3.6.

Proposition 3.6 (Maximum Principle for $\Delta^{G}$ ). Let $\psi \in \mathcal{D}_{G}$ denote an admissible function which is superharmonic on $\Omega \subset G$ so that $\Delta^{G} \psi(x) \leq 0$ for all $x \in \Omega$. Then $\psi$ has no local interior minimum in $\Omega$ : If there is an interior point $p \in \Omega$ such that $\psi(p) \leq \psi(x)$ for all $x$ in a neighbourhood $\omega(p)$ of $p$ in $\Omega$, then $\psi(x)=\psi(p)$ for all $x \in \omega(p)$. In a similar way subharmonicity rules out the existence of interior maxima.

Proof. At any given interior edge point $w \in e \in E$ this follows from the usual maximum principle (for the double derivative with respect to arclength along the edge). Suppose then that $p$ is a vertex, and that $\psi(p) \leq \psi(x)$ for all $x \in \omega(p) \subset Y_{p}$. Along every arclength parametrized edge $\gamma_{i}(s)$ in $Y_{p}$ we therefore have

$$
\psi^{\prime}\left(\gamma_{i}(0)\right) \geq 0
$$


From condition (3.2) we conclude, that

$$
\psi^{\prime}\left(\gamma_{i}(0)\right)=0
$$

Hence along every edge (via superharmonicity of $\psi$ there) in $\omega(p)$

$$
\psi^{\prime}\left(\gamma_{i}(s)\right)=\int_{0}^{s} \psi^{\prime \prime}\left(\gamma_{i}(t)\right) d t \leq 0
$$

This contradicts the assumption $\psi(x) \geq \psi(p)$ for all $x \in \omega(p)$ unless $\psi(x)=\psi(p)$ for all $x \in \omega(p)$, which is what we wanted to conclude.

In the last section of the present paper, which is concerned with the capacities of minimal webs, we shall also need the following version of the maximum principle, which is proved along the same lines of reasoning as above.

Proposition 3.7 (Boundary point version). Let $\psi \in \mathcal{D}_{G}$ denote an admissible function which is subharmonic on a precompact open domain $\Omega \subset G$, so that $\Delta^{G} \psi(x) \geq 0$ for all $x \in \Omega$. Suppose there exists a point $x_{0} \in \partial \Omega$ at which

$$
\psi^{\prime}\left(\gamma_{i}(0)\right)=0 \text { for all } \gamma_{i} \subset Y_{x_{0}} \cap \Omega .
$$

Then $\psi(x)=\psi\left(x_{0}\right)$ for all $x \in \Omega$.

\section{Minimal immersions}

Proposition 4.1. Let $f \in \mathcal{D}_{G}$. For each vertex $p$ there exists a minimal isometric immersion of the $p$-centered star space $Y_{p}$ into a Euclidean space $\mathbb{R}^{n}$ (of sufficiently high dimension) and a smooth function $U: \mathbb{R}^{n} \rightarrow \mathbb{R}$ such that $f$ is the restriction of $U$ to $Y_{p}$, i.e. $f=U_{\left.\right|_{Y_{p}}}$.

Proof. This follows from solving the relevant linear system of equations for the cofficients in the Taylor series expansion of $U$ at the point $p$.

Remark 4.2. Proposition 4.1 raises the interesting question of obtaining conditions under which a given complete metric graph $G$ admits an isometric minimal immersion (or embedding) into some Euclidean space or, say, into a given space form of constant curvature. We shall not pursue this question further here. If such an immersion exists, then it is probably not unique in general - it may be quite flexible in the same way as exemplified by the families of associated pairwise isometric minimal surfaces in $\mathbb{R}^{3}$. Concerning graphs on surfaces, the combinatorial (non-metric) embedding problem is thoroughly covered in $[\mathrm{MT}]$.

Proposition 4.3. Suppose $G$ is a vertex minimal isometric immersion in $N^{n}$ and let $\phi$ denote a smooth function on $N^{n}$. Then the restriction $f$ of $\phi$ to $G$ is an admissible function on $G$,

$$
f=\phi_{\left.\right|_{G}} \in \mathcal{D}_{G} \text {. }
$$


Proof. The function $\phi$ is clearly smooth on the (open) edges of $G$. At any given vertex $p$ we have, using again the parametrized star space $Y_{p}$

$$
\phi^{\prime}\left(\gamma_{i}(0)\right)=\left\langle\nabla^{N} f, \dot{\gamma}_{i}(0)\right\rangle_{N},
$$

so that by vertex-minimality at $p$

$$
\begin{aligned}
\sum_{i=1}^{m(p)} \phi^{\prime}\left(\gamma_{i}(0)\right) & =\left\langle\nabla^{N} f, \sum_{i=1}^{m(p)} \dot{\gamma}_{i}(0)\right\rangle_{N} \\
& =\left\langle\nabla^{N} f, 0\right\rangle_{N}=0 .
\end{aligned}
$$

Lemma 3.1 then applies and gives the result.

\section{Divergence theorems}

A vector field $X$ on $G$ is a (smooth) choice of tangent vector at each point of every edge. A vector field is thus $m(p)$-valued at any given vertex $p$. Along the 1-dimensional interior of every edge $e_{i}$ in the $p$-centered star space $Y_{p}$ a given vector field $X$ is integrable and may thus be considered as the gradient of a smooth function $f_{i}$ on $e_{i}$ in $Y_{p}$ :

$$
X_{\left.\right|_{e_{i}}}=\nabla^{e_{i}}\left(f_{i}\right)=f_{i}^{\prime}\left(\gamma_{i}(s)\right) \cdot \dot{\gamma}_{i}(s) \text { for some } f_{i} \in \mathcal{D}_{G} \quad .
$$

Note that $f_{i}\left(\gamma_{i}(s)\right)$ is defined only modulo arbitrary constants of integration and that the sign of $f_{i}^{\prime}\left(\gamma_{i}(s)\right)$ depends on the parametrization of $\gamma_{i}$ in $Y_{p}: f_{i}^{\prime}\left(\gamma_{i}(s)\right)=\left\langle X, \dot{\gamma}_{i}(s)\right\rangle_{G}$. The inner product $\langle., .\rangle_{G}$ stems from the geometrization of $G$. We will say that $X$ is admissible in $G$ if for every vertex $p$ we have

$$
\sum_{i=1}^{m(p)} f_{i}^{\prime}\left(\gamma_{i}(0)\right)=0
$$

where $f_{i}\left(\gamma_{i}(s)\right)$ is any (local integral) function representing $X$ on the star space $Y_{p}$.

Conversely, suppose $f$ is an admissible smooth function on $G$. Then the gradient of $f$ is the vector field $(m(p)$-valued at the vertex $p)$

$$
\nabla^{G}(f)=f^{\prime}(\gamma(s)) \cdot \dot{\gamma}(s) \text { along every edge } e=\gamma([0, L(e)]) .
$$

The gradient vector field is clearly admissible in $G$ because of equation (3.2).

Definition 5.1. Let $X$ be a smooth admissible vector field on $G$ as defined above with the local integrals $f_{i}$ on the edges of $Y_{p}$. By suitable choice of integration constants we may assume without lack of generality that the values of $f_{i}$ agree at $p$, and that $f$ is a single-valued smooth function on $Y_{p}$ which agrees with $f_{i}$ along the edges emanating from $p$. Then we define

$$
\operatorname{div}(X)=\operatorname{div}\left(\nabla^{G} f\right)=\Delta^{G}(f)
$$


Remark 5.2. This 'definition-by-local-construction' only depends on the vector field $X$ and not on the local representing integrals $f$ nor $f_{i}$.

With this definition the familiar divergence theorems hold true. Indeed, let us consider a domain $\Omega$ in $G$, i.e. $\Omega$ is a precompact, open, connected subset of $G$ with boundary denoted by $\partial \Omega$, and let $X$ denote a smooth admissible vector field on $G$.

Theorem 5.3 (Divergence theorem).

$$
\int_{\Omega} \operatorname{div}(X) d V=\sum_{\partial \Omega}\langle X, \nu\rangle_{G} .
$$

Here $d V$ denotes the measure on the graph induced from the geometrization of $G$. The vectors $\nu$ are the outward (from $\Omega$ ) pointing unit tangent vectors of the closed segments of edges in $\bar{\Omega}$ at the respective points of intersection with $\partial \Omega$. If $\partial \Omega$ contains a vertex from $G$, then $\nu$ and $X$ may be multi-valued at this point in $\partial \Omega$, in which case the sum has a contribution from each of the outward pointing unit tangent directions.

Proof. The theorem follows from the 'one-dimensional' divergence theorem applied to the union of open edges of $G$ in $\Omega$ together with the following observation: The contribution to the left hand side of equation (5.5) from the inner vertices of $G$ in $\Omega$ vanishes because of the balancing condition (3.2) at the 'center' of every star space.

The corresponding Green's theorems may now be stated as follows:

Theorem 5.4. Let $h, f \in \mathcal{D}_{G}$ denote smooth admissible functions on G. Then

$$
\begin{gathered}
\int_{\Omega}\left(h \Delta^{G} f+\left\langle\nabla^{G} h, \nabla^{G} f\right\rangle_{G}\right) d V=\sum_{\partial \Omega} h \cdot\left\langle\nabla^{G} f, \nu\right\rangle_{G} \quad \text { and } \\
\int_{\Omega}\left(h \Delta^{G} f-f \Delta^{G} h\right) d V= \\
\sum_{\partial \Omega}\left(h \cdot\left\langle\nabla^{G} f, \nu\right\rangle_{G}-f \cdot\left\langle\nabla^{G} h, \nu\right\rangle_{G}\right)
\end{gathered}
$$

\section{The First DiRichlet EIGENVAlue}

Much of the well known analysis of functions on domains in manifolds can be extended to domains of geometrized graphs $G$ as long as we restrict attention to admissible functions. In particular we can study the eigenfunctions of $\Delta^{G}$ in $\mathcal{D}_{G}$. The Dirichlet spectrum of $\Delta^{G}$ is purely discrete on precompact sub-webs of $G$, see e.g. [Cat] and [FT]. The so-called $R$-webs, which are defined in the following section 7 , will be our main examples of such sub-webs of $G$. 
For the Laplacian defined in Section 3 we consider the smallest eigenvalue $\lambda_{1}(\Omega)$ in the Dirichlet spectrum of any given precompact domain $\Omega$ in $G$, i.e. $\lambda_{1}$ is the smallest real number for which the following problem has a non-zero solution $u \in \mathcal{D}_{\Omega}$ :

$$
\left\{\begin{array}{ccl}
\Delta^{G} u(x)+\lambda_{1} u(x) & =0 & \text { at all points } x \in \Omega \\
u(x) & =0 & \text { at all points } x \in \partial \Omega .
\end{array} .\right.
$$

Proposition 6.1. The first eigenfunction is nowhere zero in the interior of the domain and has multiplicity 1.

Proof. This follows almost verbatim from the proof of the corresponding statement for domains in Riemannian manifolds together with the maximum principle, see e.g. [C2].

A beautiful observation due to Barta concerning the estimation of first eigenvalues of precompact domains on manifolds can therefore be extended to precompact domains of geometrized graphs as follows (cf. also [OU], [Fr]).

Theorem A ([B] $)$. Let $\Omega$ denote a given precompact domain in $G$ and let $f \in \mathcal{D}_{\Omega}$ be any admisssible function on $\Omega$, which satisfies $f_{\left.\right|_{\Omega}}>0$ and $f_{\left.\right|_{\partial \Omega}}=0$. Then the first eigenvalue $\lambda_{1}$ of the Dirichlet problem on $\Omega$ is bounded as follows

$$
\inf _{\Omega}\left(\frac{\Delta^{G} f}{f}\right) \leq-\lambda_{1} \leq \sup _{\Omega}\left(\frac{\Delta^{G} f}{f}\right) .
$$

If $[=]$ occurs in any one of the two inequalities in (6.2), then $f$ is an eigenfunction for $\Omega$ corresponding to the eigenvalue $\lambda_{1}$.

Proof. Let $\phi$ be an eigenfunction for $\Omega$ corresponding to $\lambda_{1}$. Then we may assume without lack of generality that $\phi_{\left.\right|_{\Omega}}>0$ and $\phi_{\left.\right|_{\partial \Omega}}=0$. If we let $h$ denote the difference $h=\phi-f$, then

$$
\begin{aligned}
-\lambda_{1}=\frac{\Delta^{G} \phi}{\phi} & =\frac{\Delta^{G} f}{f}+\frac{f \Delta^{G} h-h \Delta^{G} f}{f(f+h)} \\
& =\inf _{\Omega}\left(\frac{\Delta^{G} f}{f}\right)+\sup _{\Omega}\left(\frac{f \Delta^{G} h-h \Delta^{G} f}{f(f+h)}\right) \\
& =\sup _{\Omega}\left(\frac{\Delta^{G} f}{f}\right)+\inf _{\Omega}\left(\frac{f \Delta^{G} h-h \Delta^{G} f}{f(f+h)}\right) .
\end{aligned}
$$

Here the supremum, $\sup _{\Omega}\left(\frac{f \Delta^{G} h-h \Delta^{G} f}{f(f+h)}\right)$ is necessarily positive since

$$
f(f+h)_{\left.\right|_{\Omega}}>0
$$

and since by Green's second formula (5.7) in Theorem 5.4 we have

$$
\int_{\Omega}\left(f \Delta^{G} h-h \Delta^{G} f\right) d V=0
$$




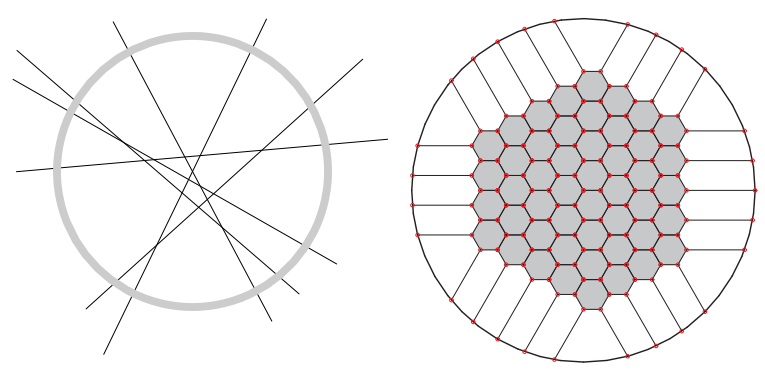

Figure 6. Any finite system of intersecting straight lines in the plane (with a vertex at each intersection point) is a minimal web in $\mathbb{R}^{2}$. Portions of regular hexagonal 'fillings' also generate minimal webs.

For the same reason, the infimum, $\inf _{\Omega}\left(\frac{f \Delta^{G} h-h \Delta^{G} f}{f(f+h)}\right)$ is necessarily negative. This gives the first part of the theorem. If equality occurs, then $\left(f \Delta^{G} h-h \Delta^{G} f\right)$ vanishes identically on $\Omega$, so that $-\lambda_{1}(\Omega)=\frac{\Delta^{G} f}{f}$, which gives the last part of the statement.

Along the same lines of reasoning we can establish Rayleigh's Theorem, the Max-Min theorem and the Domain Monotonicity (of eigenfunctions) almost verbatim from the classical analysis, see e.g [C1], [C2].

\section{EXTRINSIC DisTANCE ANALYSIS ON MINIMAL WEBS}

We let $G$ be a complete immersed minimal web in an ambient Riemannian manifold $\left(N^{n}, h\right)$ with bounded sectional curvatures ( i.e. $K_{N} \leq b$ or $K_{N} \geq b$ respectively, for some $b$ ). Let $p$ denote a point in $G$ - not necessarily a vertex point - and let $B_{R}(p)$ denote the geodesic distance ball of radius $R$ and center $p$ in $\left(N^{n}, h\right)$ :

$$
B_{R}(p)=\left\{x \in N \mid \operatorname{dist}_{N}(p, x) \leq R\right\} .
$$

The distance from $p$ will be denoted by $r$ so that $r(x)=\operatorname{dist}_{N}(p, x)$ for all $x \in N$. In particular $G$ inherits the function $r_{\left.\right|_{G}}$, which will also be denoted by $r$.

Since we shall need differentiability of certain distance dependent functions $F \circ r$ in our analysis below, we will assume that the balls under consideration are always diffeomorphic to a standard Euclidean ball via the exponential map in $N^{n}$ from the center point. This is guaranteed by bounding the radius as follows:

$$
R<\frac{\pi}{2 \sqrt{k}} \text { and } R<i_{N}(p), \quad \text { where }
$$

(1) $k=\sup _{x \in B_{R}(p)}\left\{K_{N}(\sigma) \mid \sigma\right.$ is a two-plane in $\left.T_{x} B_{R}(p)\right\}$, 

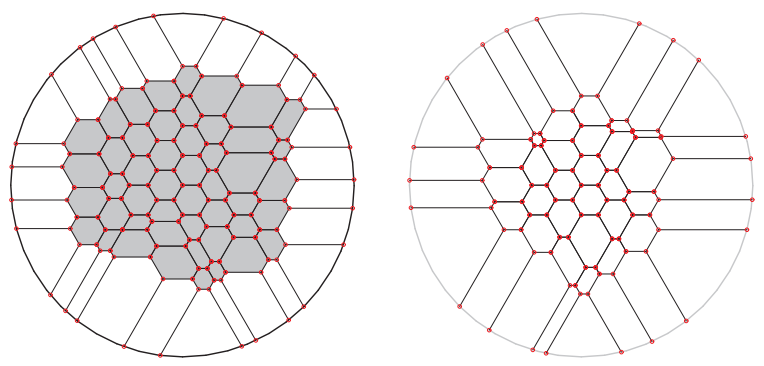

Figure 7. Examples of hexagonal minimal $R$-webs in $\mathbb{R}^{2}$.

(2) $K_{N}(\sigma)$ denotes the sectional curvature in $N^{n}$ of the 2-plane $\sigma$,

(3) $\frac{\pi}{2 \sqrt{k}}=\infty$ if $\mathrm{k} \leq 0$, and

(4) $i_{N}(p)=$ the injectivity radius of $\exp _{p}$ in $N$.

The intersection of the interior of a regular ball $B_{R}(p)$ with $G$ will be called an extrinsic minimal $R$-web of the web $G$, and will be denoted by

$$
W_{R}(p)=B_{R}(p) \cap G
$$

The geodesic balls $B_{R}(p)$ are strongly convex as follows directly from [Sa, proof of Theorem 5.3]. Thus any two points in $B_{R}(p)$ can be joined by a unique minimal geodesic which is completely contained in the ball $B_{R}(p)$. Therefore, when the boundary $\partial B_{R}(p)$ meets a (geodesic) edge of $W_{R}(p)$, then the prolongation of this geodesic intersects the boundary transversally.

Without lack of generality we may and do add vertices to $W_{R}(p)$ at these intersections with the boundary of the ambient ball $B_{R}(p)$, so that $W_{R}$ becomes the image of a web in its own right with a well defined vertex set boundary $\partial W_{R}(p)$. We refer to Figures 6,7 , and 8 for examples indicating how to construct a variety of $R$-webs in the plane.

We then obtain the (2.nd order) comparison theory for the $F$-modified distance functions on extrinsic $R$-webs of minimal webs by first specializing the corresponding theory for minimal submanifolds (as developed in e.g. $[\mathrm{JK}],[\mathrm{P}],[\mathrm{MP} 1]-[\mathrm{MP} 3]$, and $[\mathrm{MM}]$ ) to the 1dimensional case of geodesics and then secondly by generalizing this to minimal webs as follows.

Proposition 7.1. Let $G \subset N^{n}$ denote a minimal web in $N^{n}$ (resp. the image of an isometric minimal immersion of $G$ into $N^{n}$ ). Let $F$ denote a smooth real function on $\mathbb{R}$, such that the function $F \circ r$ is an admissible function on $G$ within an extrinsic web $W_{R}$ of $G$. Suppose 
further that

$$
\left\{\begin{array}{cl}
K_{N} & \leq b \text { for some } b \in \mathbb{R}, \text { and that } \\
\frac{d}{d r} F(r) & \geq 0 \text { for all } r \in[0, R]
\end{array}\right.
$$

and let $Z_{F, b}(r)$ denote the function

$$
Z_{F, b}(r)=F^{\prime \prime}(r)-F^{\prime}(r) h_{b}(r),
$$

where the function $h_{b}(r)$ denotes the mean curvature of the geodesic sphere $\partial B_{r}^{b, n}$ of radius $r$ in the space form $\mathbb{K}^{n}(b)$ of constant curvature b. Specifically

$$
h_{b}(r)=\left\{\begin{array}{ll}
\sqrt{b} \cot (\sqrt{b} r), & \text { if } b>0 \\
1 / r & \text { if } b=0 \\
\sqrt{-b} \operatorname{coth}(\sqrt{-b} r) & \text { if } b<0
\end{array} .\right.
$$

Along the interior of every arclength parametrized geodesic edge $\gamma(s)$ of the web $W_{R}$ we then have for all $r=r(\gamma(s))$ :

$$
\Delta^{G}(F \circ r)_{\left.\right|_{\gamma(s)}} \geq Z_{F, b}(r) \cdot\left\langle\nabla^{N} r, \dot{\gamma}(s)\right\rangle_{N}^{2}+F^{\prime}(r) h_{b}(r) .
$$

At a given vertex $p$ in $W_{R}$ with emanating edges $\gamma_{i}(s)$ (in the corresponding $p$-centered star space $Y_{p}$ ) we get for $r=r(p)$ :

$$
\begin{aligned}
\Delta^{G}(F \circ r)_{\left.\right|_{p}} \geq Z_{F, b}(r) & \cdot\left(\frac{1}{m(p)} \sum_{i=1}^{m(p)}\left\langle\nabla^{N} r, \dot{\gamma}_{i}(0)\right\rangle_{N}^{2}\right) \\
& +F^{\prime}(r) h_{b}(r) .
\end{aligned}
$$

Proof. Along the interior of each geodesic edge in $G$ this follows directly from the result for minimal submanifolds (in casu geodesics) in $N^{n}$ on the basis of standard index comparison theory for Jacobi fields along the distance realizing minimal geodesics from $p$, see e.g. [MM]. The Laplace inequality at vertices is then obtained by averaging the Laplace inequalities (7.7) over the directions in $Y_{q}$ emanating from $q$.

In particular we note the following consequences

Corollary 7.2. If precisely one of the inequalities in the asumptions (7.4) is reversed, then the inequalities (7.7) and (7.8) are likewise reversed.

Corollary 7.3. If at least one of the inequalities in the assumptions (7.4) is actually an equality (i.e. $N=\mathbb{K}^{n}(b)$ or $F(t)=$ constant), then the inequalities (7.7) and (7.8) are equalities as well. 


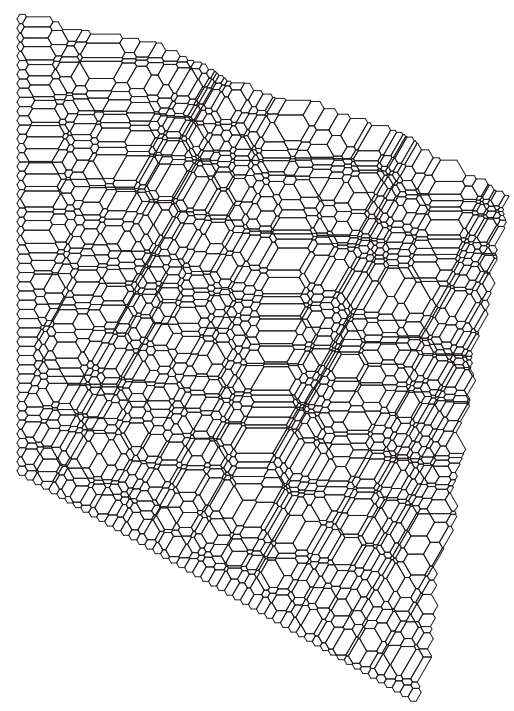

Figure 8. A foam-like wedge portion of a hexagonal web in $\mathbb{R}^{2}$.

\section{Minimal $R$-Webs IN SPACE FORMS}

If we consider functions $F$ satisfying $Z_{F, b}(r)=0$ for all $r \in[0, R]$, and if we furthermore assume that $N=\mathbb{K}^{n}(b)$, then we get the following results for minimal webs in space forms.

Proposition 8.1. Let $G \subset \mathbb{K}^{n}(b)$ denote a minimal web in $\mathbb{K}^{n}(b)$. In any extrinsic web $W_{R}$ of $G$ the following identities hold for all $r \in[0, R]$ :

$$
\begin{gathered}
\Delta^{G} \cos (\sqrt{b} r)=-b \cos (\sqrt{b} r) \quad \text { for } b>0, \\
\Delta^{G}\left(\frac{1}{2} r^{2}\right)=1 \quad \text { for } b=0 \quad \text { and } \\
\Delta^{G} \cosh (\sqrt{-b} r)=-b \cosh (\sqrt{-b} r) \quad \text { for } b<0 .
\end{gathered}
$$

Proof. In all 3 cases the function $Z_{F, b}(r)$ vanishes identically, so the statements follow from Corollary 7.3 and Proposition 7.1.

8.1. An exact first Dirichlet eigenvalue. With reference to Theorem A in section 6 we thus get from equation (8.1) the exact first Dirichlet eigenvalue for minimal webs in any hemisphere:

Corollary 8.2. Let $G \subset \mathbb{K}^{n}(b)$ denote a minimal web in the sphere of constant positive sectional curvature $b$. Then the first Dirichlet eigenvalue of any extrinsic minimal $\left(\frac{\pi}{2 \sqrt{b}}\right)-$ web of $G$ is

$$
\lambda_{1}\left(W_{\frac{\pi}{2 \sqrt{b}}}\right)=b .
$$


8.2. An exact mean exit time function. Furthermore, referring to Remark 3.5 we may consider the Brownian motion on a given minimal web as a limit process of the random walk on the subdivided and scaled combinatorial web. The discrete combinatorial Laplacian (the difference operator defined by Dodziuk in [Dod2]) as well as the smooth Laplacian (on Riemannian manifolds) both give rise to a theory of diffusion on the corresponding geometric background - via the heat equation and its kernel solutions, see e.g. [Dy], [CLY], [M3, MM]. Accordingly we define the mean exit time functions $E_{R}$ for the Brownian motion ('driven' by the operator $\Delta^{G}$ ) on minimal webs $G$ - in casu extrinsic minimal webs $W_{R}$ in $G$ - as follows:

Definition 8.3. Let $W_{R}$ denote an extrinsic $R$-web of a minimal web $G$ in an ambient Riemannian manifold $N^{n}$. Then the mean exit time function $E_{R}(x)$ for the Brownian motion on $W_{R}$ from the point $x$ is the unique continuous solution in $\mathcal{D}_{W_{R}}$ to the following boundary value Poisson problem on $W_{R}$

$$
\left\{\begin{array}{ll}
\Delta^{G} E_{R}(x) & =-1 \text { at all } x \in W_{R}, \text { and } \\
E_{R}(x) & =0 \text { at all } x \in \partial W_{R}
\end{array} .\right.
$$

Using Proposition 8.1, equation (8.2), we then obtain the following result:

Theorem 8.4. Let $W_{R}(p)$ denote an extrinsic $p$-centered web of a minimal web $G$ in $\mathbb{R}^{n}$. Then the mean exit time from any given starting point $x \in W_{R}$ is

$$
E_{R}(x)=\frac{1}{2}\left(R^{2}-r^{2}(x)\right),
$$

where $r(x)$ as usual denotes the Euclidean distance in $\mathbb{R}^{n}$ of $x$ from $p$.

Remark 8.5. A somewhat surprising interpretation of this result is the following. Consider a maze in $\mathbb{R}^{n}$ constructed in such a way that the underlying graph is an extrinsic $R$-web of a minimal web. The theorem roughly says that if you get lost in the maze at some place with Euclidean distance $r$ from $p$ then by performing a Brownian motion in the maze, then (in the mean) you will get out of the maze as quickly as if you had performed a Brownian motion on a straight line segment of length $2 R$ starting at distance $r$ from the center of the segment.

\section{Minimal Webs in nOnCONStant CURVATURE}

In ambient spaces with varying (but bounded) curvature we expect the equalities of the above space form results to be replaced by suitable inequalities. Since $\nabla^{N} r$ and $\dot{\gamma}_{i}(s)$ are both unit vectors, we certainly have the following basic inequality which will be instrumental for our applications: 


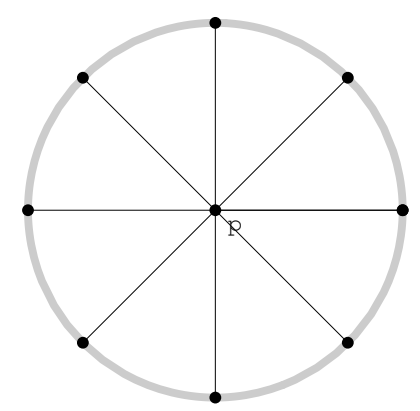

Figure 9. An extrinsic minimal star web $W_{2}^{*}(p)$ of radius 2 in the Euclidean plane.

$$
\left\langle\nabla^{N} r, \dot{\gamma}_{i}(s)\right\rangle_{N}^{2} \leq 1
$$

We note that if equality holds in 9.1 for all edges $\gamma_{i}$ in a given web $W_{R}(p)$, then $\nabla^{N} r=\dot{\gamma}_{i}(s)$ and therefore the web is a star web of radius $R$ consisting of $m(p)$ geodesic line graphs each of length $R$ emanating from $p$, see Figure 9.

From these observations together with Proposition 7.1 (equations (7.7) and (7.8)) we then get the following comparison inequalities and corresponding rigidity statements:

Proposition 9.1. We consider a minimal web $G$ in $N^{n}$, and let $W_{R}(p)$ denote an extrinsic minimal web of $G$. (In the following we let $K_{N} \leq b$ be the shorthand notation for the assumption $K_{N}(\sigma) \leq b$ for every 2 -plane $\sigma$ in $N^{n}$. Further we let, for example, $F^{\prime}(r) \geq 0$ represent that assumption for all $r \in[0, R]$ and similarly for $Z_{F, b}(r) \leq 0$.) Then the following inequalities hold true at every point $x \in W_{R}(p)$ :

$$
\begin{aligned}
& \left(\begin{array}{rl}
K_{N} & \leq b \\
F^{\prime}(r) & \geq 0 \\
Z_{F, b}(r) & \leq 0
\end{array}\right) \Longrightarrow \Delta^{G}(F \circ r)_{\left.\right|_{x}} \geq F^{\prime \prime}(r)_{\left.\right|_{x}} \\
& \left(\begin{array}{rl}
K_{N} & \leq b \\
F^{\prime}(r) & \leq 0 \\
Z_{F, b}(r) & \geq 0
\end{array}\right) \Longrightarrow \Delta^{G}(F \circ r)_{\left.\right|_{x}} \leq F^{\prime \prime}(r)_{\left.\right|_{x}} \\
& \left(\begin{array}{rl}
K_{N} & \geq b \\
F^{\prime}(r) & \geq 0 \\
Z_{F, b}(r) & \geq 0
\end{array}\right) \Longrightarrow \Delta^{G}(F \circ r)_{\left.\right|_{x}} \leq F^{\prime \prime}(r)_{\left.\right|_{x}}
\end{aligned}
$$




$$
\left(\begin{array}{rl}
K_{N} & \geq b \\
F^{\prime}(r) & \leq 0 \\
Z_{F, b}(r) & \leq 0
\end{array}\right) \Longrightarrow \Delta^{G}(F \circ r)_{\left.\right|_{x}} \geq F^{\prime \prime}(r)_{\left.\right|_{x}} \quad .
$$

If $Z_{F, b}(r) \neq 0$ almost everywhere in $[0, R]$ and if $\Delta^{G}(F \circ r)_{\left.\right|_{x}}=$ $F^{\prime \prime}(r)_{\left.\right|_{x}}$ almost everywhere in $[0, R]$, then $W_{R}(p)$ is a star web of radius $R$ from $p$.

For the applications below we need to study those modified distance functions for which the right hand sides of the Laplace inequalities in (9.2)-(9.5) are constants. Specifically we have the following immediate consequences of Proposition 9.1:

Corollary 9.2. Let $F$ denote a function with $F^{\prime}(r)=c-r$ for some constant $c \in \mathbb{R}$ (so that $F^{\prime \prime}(r)=-1$ ). Then we get the following Laplace inequalities for minimal extrinsic webs $W_{R}$ in $N$ :

$$
\left\{\begin{array}{l}
\text { If } c \geq R, \text { then } \Delta^{G}(F \circ r) \geq-1 . \\
\text { If } K_{N} \leq b \leq 0 \text { and } c \leq 0, \text { then } \Delta^{G}(F \circ r) \leq-1 . \\
\text { If } K_{N} \geq b \geq 0 \text { and } c=0, \text { then } \Delta^{G}(F \circ r) \geq-1 .
\end{array}\right.
$$

If $(c \neq 0$ or $b \neq 0)$ and if $\Delta^{G}(F \circ r)=-1$ almost everywhere in $[0, R]$, then $W_{R}$ is a star web of radius $R$ from $p$.

Proof. Since the sign discussion for $F^{\prime}(r)$ is quite obvious, we only have to consider the sign of $Z_{F, b}(r)=-1-(c-r) h_{b}(r)$. We get for all $r \in[0, R]$ : If $c \leq 0$ and $b \leq 0$, then $Z_{F, b}(r) \geq 0$; If $c \geq 0$ and $b \geq 0$, then $Z_{F, b}(r) \leq 0$; If $c \geq R$, then $Z_{F, b}(r) \leq 0$ for all $b$. The Corollary then follows directly from Proposition 9.1. In all cases $Z_{F, b}(r) \neq 0$ unless $b=0$, so that the rigidity conclusion holds true as well.

Corollary 9.3. Let $F$ denote a function with $F^{\prime}(r)=c$ for some constant $c \in \mathbb{R}$ (so that $F^{\prime \prime}(r)=0$ ). Then we get the following Laplace inequalities for minimal extrinsic webs $W_{R}$ in $N$ :

$$
\left\{\begin{array}{l}
\text { If } c \geq 0, \text { then } \Delta^{G}(F \circ r) \geq 0 . \\
\text { If } c \leq 0, \text { then } \Delta^{G}(F \circ r) \leq 0 .
\end{array}\right.
$$

If $c \neq 0$ and if $\Delta^{G}(F \circ r)=0$ almost everywhere in $\left.] 0, R\right]$, then $W_{R}$ is a star web of radius $R$ from $p$.

Proof. The sign discussions for $F^{\prime}(r)$ and $Z_{F, b}(r)=-c h_{b}(r)$, respectively, is now obvious. We get for all $r \in] 0, R]$ : If $c \leq 0$, then $Z_{F, b}(r) \geq 0$; If $c \geq 0$, then $Z_{F, b}(r) \leq 0$; The Corollary again follows from Proposition 9.1. For $c \neq 0$ we get $Z_{F, b}(r) \neq 0$, so that the rigidity conclusion again holds true. 


\subsection{Eigenvalue inequalities.}

Theorem 9.4. Let $G \subset N^{n}$ denote a minimal web in an ambient Riemannian manifold $N^{n}$. Then the first Dirichlet eigenvalue of any extrinsic minimal $R$-web $W_{R}$ of $G$ satisfies

$$
\lambda_{1}\left(W_{R}\right) \geq\left(\frac{\pi}{2 R}\right)^{2},
$$

and equality is attained if and only if $W_{R}$ is a star web.

Proof. We use Barta's Theorem A (from section 6) on the test function $F(r)=\cos \left(\frac{\pi}{2 R} r\right)$. Let $b$ denote the supremum

$$
b=\sup _{x \in B_{R}(p)}\left\{K_{N}(\sigma) \mid \sigma \text { is a two-plane in } T_{x} B_{R}(p)\right\} .
$$

In view of Proposition 9.1 equation (9.3) we only need to show, that $Z_{F, b}(r)>0$ for all $\left.\left.r \in\right] 0, R\right]$. But this is a consequence of the following equivalent inequalities:

$$
\begin{aligned}
Z_{F, b}(r) & >0 \\
-F^{\prime}(r) h_{b}(r) & >F^{\prime \prime}(r) \\
h_{b}(r)\left(\frac{\pi}{2 R}\right) \sin \left(\frac{\pi}{2 R} r\right) & >\left(\frac{\pi}{2 R}\right)^{2} \cos \left(\frac{\pi}{2 R} r\right) \\
h_{b}(r) & >\left(\frac{\pi}{2 R}\right) \cot \left(\frac{\pi}{2 R} r\right) \\
h_{b}(r) & >h_{\frac{\pi^{2}}{4 R^{2}}}(r) .
\end{aligned}
$$

Indeed, the last inequality follows from the fact that the mean curvature function $h_{b}(r)$ is a strictly decreasing function of $b$ for every fixed $r \leq$ $R$ together with the general assumption that $R<\frac{\pi}{2 \sqrt{b}}$, so that $b<$ $\frac{\pi^{2}}{4 R^{2}}$. We conclude that

$$
\Delta^{G} F(r) \leq F^{\prime \prime}(r)=-\left(\frac{\pi}{2 R}\right)^{2} F(r)
$$

The result then follows from Barta's second inequality in (6.2). Since $Z_{F, b}(r)>0$, the equality statement follows from the rigidity conclusion of Proposition 9.1.

Remark 9.5. In view of the inequality $\left(\frac{\pi}{2 R}\right)^{2}>b$, we get $\lambda_{1}\left(W_{R}\right)>$ $b$. This is, of course, only interesting when $b>0$, in which case (9.8) should be compared with Corollary 8.2. It is also informative to compare the $R$-web-eigenvalue in (9.8) with the first Dirichlet eigenvalue of a geodesic ball of radius $R$ in $\mathbb{R}^{m}$ :

$$
\lambda_{1}\left(B_{R}^{0, m}\right)=\left(\frac{j_{k}}{R}\right)^{2}>\left(\frac{\pi}{2 R}\right)^{2},
$$

where $j_{k}$ is the smallest positive zero of the Bessel function $J_{k}$ of order $k=\frac{1}{2}(m-2)$. (Here $j_{0} \simeq 2.405$ and $j_{k} \sim k \sim \frac{1}{2} m$ for $m \rightarrow \infty$.) 
9.2. Mean exit time inequalities. The mean exit time function $F$ from the extrinsic $R$-web of a minimal web in $\mathbb{R}^{n}$ satisfies $Z_{F, 0}=0$ and $F^{\prime}(r) \leq 0$ for all $r \in[0, R]$, see Definition 8.3 in combination with the $R$-web analysis above. In consequence we have the following inequalities.

Theorem 9.6. Let $W_{R}(p)$ denote an extrinsic minimal web of a minimal web $G$ in a Riemannian manifold $N^{n}$. The sectional curvatures of the ambient space are denoted by $K_{N}$. Then the mean exit time $E_{R}(x)$ from the point $x$ in $W_{R}$ satisfies the inequalities:

$$
\left\{\begin{array}{l}
E_{R}(x) \geq \frac{1}{2}\left(R^{2}-r^{2}(x)\right) \text { if } K_{N}(\sigma) \geq 0 \text { for all } \sigma \\
E_{R}(x) \leq \frac{1}{2}\left(R^{2}-r^{2}(x)\right) \text { if } K_{N}(\sigma) \leq 0 \text { for all } \sigma
\end{array} .\right.
$$

If the sectional curvatures $K_{N}(\sigma)$ are bounded strictly away from 0 in either of the two cases in (9.12), then the corresponding mean exit time function $E_{R}(x)$ is also bounded strictly (with strict inequalities) by the comparison function $\frac{1}{2}\left(R^{2}-r^{2}(x)\right)$, unless the web $W_{R}(p)$ is a star web of radius $R$.

In case of a positively curved ambient space we shall also need - in Section 11 - an upper bound on the mean exit time. A rough estimate is the following:

Theorem 9.7 (See e.g. [M2], [MP3]). Suppose that $K_{N} \leq b$ for some $b>0$, then

$$
E_{R}(x) \leq \mu_{R}^{b}(r(x)) \text { for all } x \in W_{R}
$$

where

$$
\mu_{R}^{b}(r)=\frac{\cos (\sqrt{b} r)}{b \cos (\sqrt{b} R)}
$$

Proofs of Theorems 9.6 and 9.7. When inserting the comparison functions $f(r)=\frac{1}{2}\left(R^{2}-r^{2}(x)\right)$ and $f(r)=\mu_{R}^{b}(r)$, respectively, into Proposition 9.1, equation (9.3), and using $f^{\prime}(r) \leq 0, Z_{f, b}(r)=f^{\prime \prime}(r)-$ $f^{\prime}(r) h_{b}(r)=0$ for all $r$, we get in both cases (for $\left.K_{N} \leq b\right)$ :

$$
\Delta^{P} f(r(x)) \leq f^{\prime \prime}(r)_{\left.\right|_{x}} \leq-1=\Delta^{P} E_{R}(x),
$$

so that the difference function $E_{R}(x)-f(r(x))$ is subharmonic in $W_{R}$. Furthermore, the difference is certainly non-positive on the boundary $\partial W_{R}$. The Maximum Principle then implies that the difference function is non-positive in all of $W_{R}$, and this proves the two upper bounds for $E_{R}$ in (9.12) and in (9.13), respectively.

To get the lower bound on $E_{R}$ in (9.12) we proceed with the comparison function $f(r)=\frac{1}{2}\left(R^{2}-r^{2}(x)\right)$ and apply Proposition 9.1, equation (9.5), or Corollary 9.2, from which it follows that

$$
\Delta^{P} f(r(x)) \geq f^{\prime \prime}(r)_{\left.\right|_{x}}=-1=\Delta^{P} E_{R}(x) .
$$


The difference function $E_{R}(x)-f(r(x))$ is now superharmonic in $W_{R}$. Furthermore, the difference is precisely 0 on the boundary $\partial W_{R}$. The Maximum Principle then implies that the difference function is nonnegative in all of $W_{R}$, and this proves the lower bound for $E_{R}$.

If the sectional curvature bounds in (9.12) are given by strict inequalities, then in the negatively curved case we have by compactness of $B_{R}(p)$ that $K_{N}(\sigma) \leq b$ for some negative $b$. Using this value of $b$ and still $f(r)=\frac{1}{2}\left(R^{2}-r^{2}(x)\right)$ in Proposition 9.1, equation (9.3), we now obtain $Z_{f, b}(r)=f^{\prime \prime}(r)-f^{\prime}(r) h_{b}(r)>0$ for all $r$, (because $x \operatorname{coth}(x)>1$ for all $x>0$ ) so that (according to the rigidity statement in Proposition 9.1) the identity $E_{R}(x)=f(r(x))$ is only possible if $W_{R}(p)$ is a $p$-centered star web. If the sectional curvatures are bounded positively away from 0 the same conclusion follows almost verbatim from the corresponding elementary inequality $x \cot (x)<1$ for all $x \in] 0, \frac{\pi}{2}[$.

\section{ISOPERIMETRIC INEQUALITIES}

From the divergence theorem together with the Laplace inequalities of section 9 we obtain useful isoperimetric information for extrinsic minimal webs $W_{R}$ of $G$ in $N^{n}$ such as inequalities relating the measure of the boundary (the number of incoming edges to $\partial W_{R}$ ) to the measure of the web itself (the total length or mass of the edges in $W_{R}$ ). We refer to $[\mathrm{P}],[\mathrm{MP} 1]$, and $[\mathrm{MP} 2]$ for the corresponding statements for minimal submanifolds in $N^{n}$.

To facilitate the discussion and to ease the notation, we first define the radial transversality of the boundary $\partial W_{R}(p)$ as 'seen' from the center $p$.

Definition 10.1. For a given extrinsic minimal web $W_{R}(p)$ of a minimal web $G$ in $N^{n}$, the radial transversality of the boundary $\partial W_{R}(p)$ is defined by

$$
\mathrm{T}\left(\partial W_{R}\right)=\sum_{\partial W_{R}}\left\langle\nabla^{N} r, \nu\right\rangle_{N}=\sum_{\partial W_{R}}\left\langle\nabla^{G} r, \nu\right\rangle_{G},
$$

where the sum is to be taken over every out-going unit direction $\nu$ from $W_{R}$ at the boundary $\partial W_{R}$ - as in the statement of the divergence Theorem 5.3.

Definition 10.2. Two extrinsic minimal webs are concentric if they share the same center point.

The divergence theorem then gives direct estimates of the total lengths of minimal extrinsic $R$-webs in terms of the transversalities as follows.

Proposition 10.3. Let $W_{R}(p)$ and $W_{\rho}(p), R>\rho$, denote two concentric extrinsic minimal webs, with radius $R$ and $\rho$ respectively. Then the total lengths $\mathrm{L}\left(W_{R}\right)$ and $\mathrm{L}\left(W_{\rho}\right)$ of the edges in $W_{R}$ and $W_{\rho}$, respectively, 
satisfy the following inequalities (without any further assumptions on the sectional curvatures of the ambient space)

$$
\mathrm{L}\left(W_{R}\right)-\mathrm{L}\left(W_{\rho}\right) \geq(R-\rho) \cdot \mathrm{T}\left(\partial W_{\rho}\right) .
$$

In particular we get

$$
\mathrm{L}\left(W_{R}\right) \geq R \cdot m(p) \geq 2 R .
$$

If equality occurs in (10.2) or in the first inequality of (10.3) then $W_{R}$ is a $p$-centered star space of radius $R$.

Proof. We choose $c=R$ in Corollary 9.2 and let $F^{\prime}(r)=R-r$. Then $\nabla^{G}(F \circ r) \geq-1$ for all $r \in[0, R]$. Using the divergence theorem we therefore get:

$$
\begin{aligned}
\mathrm{L}\left(W_{R}-W_{\rho}\right)= & \int_{W_{R}-W_{\rho}} 1 d V \\
\geq & \int_{W_{R}-W_{\rho}}-\Delta^{G}(F \circ r) d V \\
= & -\int_{W_{R}-W_{\rho}} \operatorname{div}\left(\nabla^{G} F \circ r\right) d V \\
= & -F^{\prime}(R) \sum_{\partial W_{R}}\left\langle\nabla^{G} r, \nu\left(\partial W_{R}\right)\right\rangle_{N} \\
& +F^{\prime}(\rho) \sum_{\partial W_{\rho}}\left\langle\nabla^{G} r, \nu\left(\partial W_{\rho}\right)\right\rangle_{N} \\
= & 0+(R-\rho) \cdot \mathrm{T}\left(\partial W_{\rho}(p)\right),
\end{aligned}
$$

where $\nu\left(\partial W_{\rho}\right)$ denotes the outward pointing unit directions from $W_{\rho}$ at $\partial W_{\rho}$ and similarly, $\nu\left(\partial W_{R}\right)$ denotes the outward pointing unit directions from $W_{R}$. Since $c \neq 0$ in this setting, equality in equation (10.4) implies rigidity via Corollary 9.2.

If we do bound the sectional curvatures of the ambient space, then we have the following dual inequalities:

Theorem 10.4. Let $W_{R}(p)$ and $W_{\rho}(p), R>\rho$, denote two concentric extrinsic minimal webs, with radius $R$ and $\rho$ respectively, in an ambient space $N^{n}$ with sectional curvatures $K_{N}$. Then we have

$$
\left\{\begin{array}{l}
\mathrm{L}\left(W_{R}\right)-\mathrm{L}\left(W_{\rho}\right) \leq R \cdot \mathrm{T}\left(\partial W_{R}\right)-\rho \cdot \mathrm{T}\left(\partial W_{\rho}\right) \text { if } K_{N} \leq b \leq 0 \\
\mathrm{~L}\left(W_{R}\right)-\mathrm{L}\left(W_{\rho}\right) \geq R \cdot \mathrm{T}\left(\partial W_{R}\right)-\rho \cdot \mathrm{T}\left(\partial W_{\rho}\right) \text { if } K_{N} \geq b \geq 0
\end{array},\right.
$$

In particular we get

$$
\left\{\begin{array}{l}
\mathrm{L}\left(W_{R}\right) \leq R \cdot \mathrm{T}\left(\partial W_{R}\right) \leq R \cdot \#\left(\partial W_{R}\right) \text { if } K_{N} \leq b \leq 0 \\
\mathrm{~L}\left(W_{R}\right) \geq R \cdot \mathrm{T}\left(\partial W_{R}\right) \text { if } K_{N} \geq b \geq 0
\end{array}\right.
$$


where $\#\left(\partial W_{R}\right)$ denotes the number of outgoing directions $\nu$ (counted with multiplicities) from $W_{R}$ along the boundary $\partial W_{R}$ - this is the same as the number of edges in $W_{R}$ which have a point in common with $\partial W_{R}$. If equality occurs in one of the inequalities in (10.5) and if $b \neq 0$, then $W_{R}$ is a p-centered star web.

Proof. We now use $c=0$ and $F^{\prime}(r)=-r$. According to Corollary 9.2 we have (but here only for $b \leq 0)$ that $\nabla^{G}(F \circ r) \leq-1$ for all $r \in[0, R]$. Again the divergence theorem applies, and this time we get

$$
\begin{aligned}
\mathrm{L}\left(W_{R}-W_{\rho}\right)= & \int_{W_{R}-W_{\rho}} 1 d V \\
\leq & \int_{W_{R}-W_{\rho}}-\Delta^{G}(F \circ r) d V \\
= & -\int_{W_{R}-W_{\rho}} \operatorname{div}\left(\nabla^{G} F \circ r\right) d V \\
= & -F^{\prime}(R) \sum_{\partial W_{R}}\left\langle\nabla^{G} r, \nu\left(\partial W_{R}\right)\right\rangle_{N} \\
& +F^{\prime}(\rho) \sum_{\partial W_{\rho}}\left\langle\nabla^{G} r, \nu\left(\partial W_{\rho}\right)\right\rangle_{N} \\
= & R \cdot \mathrm{T}\left(\partial W_{R}\right)-\rho \cdot \mathrm{T}\left(\partial W_{\rho}\right),
\end{aligned}
$$

which shows the first inequality of (10.5). The other follows similarly. For $b \neq 0$ the rigidity is likewise again a consequence of Corollary 9.2.

Corollary 10.5. Let $W_{R}(p)$ denote a p-centered extrinsic minimal $R$-web in a flat ambient space $N$. Then

$$
R \cdot \#\left(\partial W_{R}\right) \geq \mathrm{L}\left(W_{R}\right)=R \cdot \mathrm{T}\left(\partial W_{R}\right) \geq R \cdot m(p) \geq 2 R .
$$

Remark 10.6. The equality \# $\left(\partial W_{R}\right)=\mathrm{T}\left(\partial W_{R}\right)$ does not by itself imply that $W_{R}$ is a star web. This follows e.g. from an inspection of the web shown in Figure 10.

\section{Capacity and transience}

We finally apply the considerations from the previous sections to estimate capacities and transience of minimal webs.

Definition 11.1. The $p$-centered annular $(\rho, R)$-web of a minimal web $G$ in $N^{n}$ is defined by $A_{\rho, R}(p)=W_{R}(p)-W_{\rho}(p)$.

The notion of capacity of a minimal annulus $A_{\rho, R}$ of $G$ in $N^{n}$ is defined as follows. 


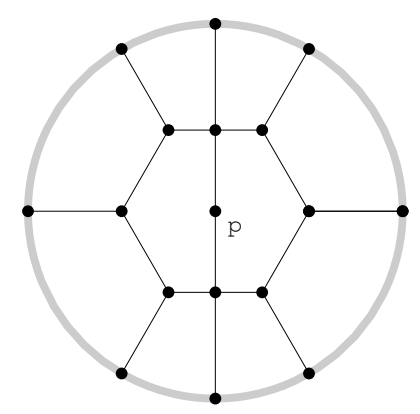

FiguRe 10. An extrinsic minimal 2 -web $W_{2}(p)$ in the Euclidean plane with $\#\left(\partial W_{2}(p)\right)=\mathrm{T}\left(\partial W_{2}(p)\right)=8$. The outer circle is $\partial B_{2}(p)$. The total length $\mathrm{L}\left(W_{2}\right)$ of $W_{2}(p)$ is 16 - in accordance with Corollary 10.5 and Remark 10.6.

Definition 11.2. Let $\Psi \in \mathcal{D}_{A_{\rho, R}}$ denote the harmonic function on $A_{\rho, R}$ which satisfies

$$
\left\{\begin{aligned}
\Delta^{G} \Psi(x) & =0 \text { for all } x \in A_{\rho, R}, \\
\Psi(x) & =0 \text { for all } x \in \partial W_{\rho} \text { and } \\
\Psi(x) & =1 \text { for all } x \in \partial W_{R} .
\end{aligned}\right.
$$

Then

$$
\begin{aligned}
\operatorname{Cap}\left(A_{\rho, R}\right) & =\sum_{\partial W_{\rho}}\left\langle\nabla^{G} \Psi, \nu\left(\partial W_{\rho}\right)\right\rangle_{G} \\
& =\sum_{\partial W_{R}}\left\langle\nabla^{G} \Psi, \nu\left(\partial W_{R}\right)\right\rangle_{G} .
\end{aligned}
$$

Remark 11.3. The latter equality in (11.2) is, of course, due to the harmonicity of $\Psi$, and is obtained via the divergence Theorem 5.3. The harmonic function equation (11.1) with the given boundary conditions is precisely the Euler-Lagrange equation for the alternative energy expression of the capacity as in the case of smooth manifolds, see e.g. [G].

We then have the following

Theorem 11.4. Let $A_{\rho, R}(p)$ denote a $p$-centered minimal annular web in an ambient manifold $N^{n}$. Then

$$
\mathrm{T}\left(\partial W_{\rho}\right) \leq(R-\rho) \cdot \operatorname{Cap}\left(A_{\rho, R}\right) \leq \mathrm{T}\left(\partial W_{R}\right) \quad .
$$

In particular, if we let $\rho$ go to 0 , we get

$$
m(p) \leq R \cdot \operatorname{Cap}\left(A_{0, R}\right) \leq \mathrm{T}\left(\partial W_{R}\right) \leq \#\left(\partial W_{R}\right) .
$$

If equality occurs in one of the inequalities in (11.3), then $A_{\rho, R}(p)$ is isometric to a star web annulus (with $\#\left(\partial W_{R}\right)$ radial edges from $\partial W_{\rho}$ to $\left.\partial W_{R}\right)$, see Figure 11. 


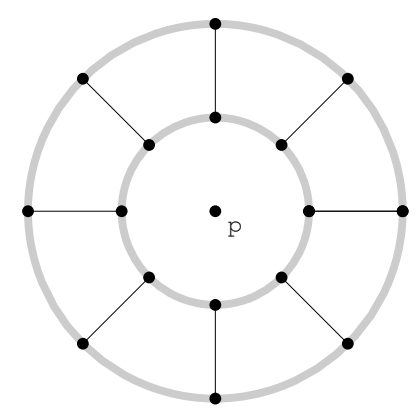

FiguRE 11. The extrinsic minimal star web annulus $A_{1,2}(p)=W_{2}^{*}(p)-W_{1}^{*}(p)$ in the Euclidean plane.

Proof. We apply Corollary 9.3 with the function $F(r)$ defined by

$$
F(\rho)=0, F(R)=1 \text {, and } F^{\prime}(r)=(R-\rho)^{-1},
$$

so that

$$
\Delta^{G} F(r)_{\left.\right|_{x}} \geq 0=\Delta^{G} \Psi(x),
$$

where $\Psi(x)$ is the solution to equation (11.1). The difference $F(r(x))-$ $\Psi(x)$ is hence a subharmonic function on $A_{\rho, R}$, and since the difference vanishes at the boundary $\partial A_{\rho, R}$, we get from the maximum principle, that

$$
F(r(x)) \leq \Psi(x) \text { for all } x \in A_{\rho, R} .
$$

In particular, along the in-boundary and out-boundary the derivatives must therefore satisfy the following inequalities

$$
\begin{gathered}
\left\langle\nabla^{G} F(r(x)), \nu\left(\partial W_{\rho}\right)\right\rangle_{G} \leq\left\langle\nabla^{G} \Psi(x), \nu\left(\partial W_{\rho}\right)\right\rangle_{G}, \\
\left\langle\nabla^{G} F(r(x)), \nu\left(\partial W_{R}\right)\right\rangle_{G} \geq\left\langle\nabla^{G} \Psi(x), \nu\left(\partial W_{R}\right)\right\rangle_{G} .
\end{gathered}
$$

It follows that

$$
\begin{aligned}
\operatorname{Cap}\left(A_{\rho, R}\right) & =\sum_{\partial W_{\rho}}\left\langle\nabla^{G} \Psi, \nu\left(\partial W_{\rho}\right)\right\rangle_{G} \\
& \geq \sum_{\partial W_{\rho}}\left\langle\nabla^{G} F(r(x)), \nu\left(\partial W_{\rho}\right)\right\rangle_{G} \\
& =F^{\prime}(\rho) \cdot \mathrm{T}\left(\partial W_{\rho}\right) \\
& =(R-\rho)^{-1} \mathrm{~T}\left(\partial W_{\rho}\right)
\end{aligned}
$$

and similarly

$$
\operatorname{Cap}\left(A_{\rho, R}\right) \leq(R-\rho)^{-1} \mathrm{~T}\left(\partial W_{R}\right) \quad .
$$

If equality occurs in (11.9) or in (11.10), then we have a corresponding equality in equation (11.8) as well. The boundary version of the maximum principle, Proposition 3.7, then applies and gives $F(r(x))=\Psi(x)$ 
for all $x \in A_{\rho, R}$. In particular all the edges in $A_{\rho, R}(p)$ must be directed radially away from $p$, and this proves the theorem.

Remark 11.5. Equalities in (11.3) do not imply that all of the $R$-web $W_{R}$ is star shaped - consider e.g. the minimal annulus $A_{1,2}(p)$ of the example $W_{2}(p)$ in Figure 10.

Definition 11.6 (Cf. [G]). A given complete metric graph $G$ is transient if there is a precompact open domain $\Omega$ in $G$, such that the Brownian motion $X_{t}$ starting from $\Omega$ does not return to $\Omega$ with probability 1, i.e. :

$$
P_{x}\left\{\omega \mid X_{t}(\omega) \in \Omega \text { for some } t>0\right\}<1,
$$

otherwise $G$ is called recurrent.

In view of Remark 3.5, and since $G=(V, E)=(G, g)$, considered as a length space continuum, is roughly isometric to $(V, d)$, considered as a combinatorial metric space with the metric $d$ induced from the length space metric $g$, we obtain: Transience of the Random Walk on $V$ with respect to $d$ is equivalent to transience of the $\Delta^{G}$-driven Brownian Motion on $G$ with respect to $g$, see e.g. [MMT].

In the literature there are several conditions for transience of manifolds and combinatorial graphs. For example, T. Lyons [Ly] obtains transience from the existence of a finite energy flow field on the graph. This, in turn, is applied by C. Thomassen in [Th] to show transience from the existence of a rooted isoperimetric profile function whose reciprocal is square integrable on the graph. The corresponding statements for manifolds are established in [LyS] by Lyons and Sullivan and in $[\mathrm{Fe}]$ by Fernandez, respectively.

The following is but one other consequence, which expresses transience in terms of capacities. The relation between these two notions is much more general than stated here (see e.g. [G], [LyS]), but we only need the following for Corollary 11.7 below.

Proposition B. A given complete minimal web $G$ in an HadamardCartan manifold is transient if for some (hence any) fixed $\rho$ we have

$$
\lim _{R \rightarrow \infty} \operatorname{Cap}\left(A_{\rho, R}\right)>0 \quad,
$$

From Theorem 11.4 we then conclude

Corollary 11.7. Let $G$ denote a complete minimal web in an Hadamard-Cartan manifold. Let $p \in G$ and let $W_{R}$ denote the $p$-centered $R$-web of $G$. Then $G$ is recurrent if

$$
\lim _{R \rightarrow \infty}\left(\frac{R}{\mathrm{~T}\left(\partial W_{R}\right)}\right)=\infty .
$$

The so-called type problem is concerned with the challenging problem of establishing sufficient (and necessary) global 'structural' conditions for a given metric graph to be transient. 
Remark 11.8. As already alluded to in subsection 2.1, Scherk's web is transient. This is shown in $[\mathrm{MMT}]$ via the finite energy flow criterion of $[\mathrm{Ly}]$.

It is to be expected that minimal webs in Hadamard-Cartan manifolds are in fact transient under conditions which should be quite mild in comparison with the intrinsic isoperimetric type conditions alluded to above. This expectation is mainly motivated by the fact that minimally immersed submanifolds (of sufficient dimension) in HadamardCartan manifolds are transient without any further conditions:

Theorem C ([MP3]). Let $P^{m}$ be a complete minimally immersed submanifold of an Hadamard-Cartan manifold $N^{n}$ with sectional curvatures bounded from above by $b \leq 0$. Then $P^{m}$ is transient if either $(b<0$ and $m \geq 2)$ or $(b=0$ and $m \geq 3)$.

However, it is not yet clear how to mold a similar condition like this 'dimensionality assumption', which in a corresponding setting would work for minimally immersed metric graphs as well.

\section{REFERENCES}

[A] C. K. Anand, Harmonic morphisms of metric graphs, In 'Harmonic morphisms, harmonic maps, and related topics' (eds.: C. K. Anand, P. Baird, E. Loubeau, and J. C. Wood), Research Notes in Mathematics Vol. 413, Chapman \& Hall (1999), 109-112.

[B] J. Barta, Sur la vibration fundamentale d'une membrane, C. R. Acad. Sci. 204 (1937), 472-473.

[Be] J. von Below, A characteristic equation associated to an eigenvalue problem on $c^{2}-$ networks, Linear Algebra Appl. 71 (1985), 309-325.

[Be2] J. von Below, Sturm-Liouville Eigenvalue Problems on Networks, Mathematical Methods in the Applied Sciences 10 (1988), 383-395.

[BH] M. R. Bridson and A Haefliger, Metric spaces of non-positive curvature, Grundlehren der Mathematischen Wissenschaften [Fundamental Principles of Mathematical Sciences] 319, Springer-Verlag, Berlin, (1999).

[BHS] A. I. Bobenko T. Hoffmann, and B. A. Springborn, Minimal surfaces from circle patterns: Geometry from combinatorics, Annals of Math. (2) 164 (2006), 231-264.

[BP] A. I. Bobenko and U. Pinkall, Discrete isothermic surfaces, J. reine angew. Math. 475 (1996), 187-208.

[BuBI] D. Burago, Y. Burago, and S. Ivanov, A Course in Metric Geometry, Graduate Studies in Mathematics, 33 American Mathematical Society (2001).

[Cat] C. Cattaneo, The spectrum of the continuous Laplacian on a graph, Monatsh. Math. 124 (1997), 215-235.

[C1] I. Chavel, Eigenvalues in Riemannian Geometry, Academic Press (1984).

[C2] I. Chavel, Riemannian Geometry: A Modern Introduction, Cambridge Tracts in Mathematics 108, Cambridge University Press (1993).

[C3] I. Chavel, Isoperimetric Inequalities, Cambridge Tracts in Mathematics 145, Cambridge University Press (2001).

[CLY] S. Y. Cheng, P. Li, and S. T. Yau, Heat equations on minimal submanifolds and their applications, Amer. J. Math. 106 (1984), 1033-1065. 
[Ch] F. R. K. Chung, Spectral Graph Theory, CBMS Regional Conference Series in Mathematics 92, American Mathematical Society (1997).

[ChY] F. Chung and S. T. Yau, Eigenvalue inequalities for graphs and convex subgraphs, Comm. Anal. Geom. 5 (1997), 575-623.

[CoG] T. Coulhon and A. Grigor'yan, Random walks on graphs with regular volume growth, GAFA, Geom. funct. anal. 8 (1998), 656-701.

[CouFL] R. Courant, K. Friedrichs, and H. Lewy, Über die partiellen Differenzengleichungen der Mathematischen Physik, Math. Ann. 100 (1928), 32-74.

[CvDS] D. M. Cvetković, M. Doob, and H. Sachs, Spectra of Graphs, Theory and Application, Academic Press (1980).

[D] E. B. Davies, Spectral Theory and Differential Operators, Cambridge studies in advanced mathematics 42, Cambridge University Press (1995).

[DL] M. M. Deza and M. Laurent, Geometry of Cuts and Metrics, Algorithms and Combinatorics 15, Springer-Verlag (1997).

[Dod1] J. Dodziuk, Finite difference approach to the Hodge theory of harmonic forms, Amer. J. Math. 98 (1976), 79-104.

[Dod2] J. Dodziuk, Difference equations, isoperimetric inequality, and transience of certain random walks, Trans. Amer. Math. Soc. 284 (1984), 787-794.

[DodKa] J. Dodziuk and L. Karp, Spectral and function theory for combinatorial Laplacian, Contemporary Math. 73 (1988), 25-40.

[DodKe] J. Dodziuk and W. S. Kendall, Combinatorial Laplacians and isoperimetric inequality, In 'From Local Times to Global Geometry, Control and Physics' (ed. K. D. Elworthy), Pitman Research Notes Math. Ser. 150 (1986), pp. 68-74.

[Dy] E. B. Dynkin, Markov processes, Springer Verlag (1965).

[E] B. Eckmann, Harmonische Funktionen und Randwertaufgaben in eiem Komplex, Comment. Math. Helv. 17 (1945), 240-255.

[EF] J. Eells and B. Fuglede, Harmonic Maps between Riemannian Polyhedra, Cambridge Tracts in Mathematics, 142, Cambridge University Press (2001).

[EP] P. Exner and O. Post, Convergence of spectra of graph-like thin manifolds, Journal of Geometry and Physics, 54 (2005), 77-115.

[Fe] J. L. Fernandez, On the existence of Green's function in a Riemannian manifold, Proc. Amer. Math. Soc. 96 (1986), 284-286.

[Fr] J. Friedman, Some geometric aspects of graphs and their eigenfunctions, Duke Math. J. 69 (1993), 487-525.

[FT] J. Friedman and J.-P. Tillich, Wave equations for graphs and the edgebased Laplacian, Pacific Journal of Mathematics 216 (2004), 229-266.

[Fu] K. Fujiwara, Eigenvalues of Laplacians on a closed Riemannian Manifold and its Nets, Proc. Amer. Math. Soc. 123 (1995), 2585-2594.

[G] A. Grigor'yan, Analytic and geometric background of recurrence and non-explosion of the Brownian motion on riemannian manifolds, Bull. (N.S.) Amer. Math. Soc. 36 (1999), 135-249.

[H] W. V. D. Hodge, The Theory and Applications of Harmonic Integrals, Cambridge at the University Press (1959).

[JK] L. P. Jorge and D. Koutroufiotis, An estimate for the curvature of bounded submanifolds, Amer. J. Math. 103 (1981), 711-725.

[Ka1] M. Kanai, Rough isometries and combinatorial approximations of geometries of non-compact Riemannian manifolds, J. Math. Soc. Japan 37 (1985), 391-413. 
[Ka2] M. Kanai, Rough isometries and the parabolicity of Riemannian manifolds, J. Math. Soc. Japan 38 (1986), 227-238.

[Ka3] M. Kanai, Analytic inequalities and rough isometries between noncompact Riemannian manifolds, In 'Curvature and topology of Riemannian manifolds' (eds. K. Shiohama, T. Sakai, and T. Sunada), Lecture Notes in Mathematics 1201 (Springer, Berlin, 1986), pp. 122-137.

[Ku1] P. Kuchment, Quantum graphs. I. Some basic structures, Waves Random Media 14 (2004), 107-128.

[Ku2] P. Kuchment, Quantum graphs. II. Some spectral properties of quantum and combinatorial graphs, J. Phys. A 14 (2005), 4887-4900.

[Lions] J. L. Lions, Problèmes aux limites dans les équations aux dérivées partielles. Séminaire de Mathématiques supérieures Montréal, Univ. Press (1962).

[Ly] T. Lyons, A simple criterion for transience of a reversible Markov chain, Ann. Probab. 11 (1983), 393-402.

[LyS] T. Lyons and D. Sullivan, Function theory, random paths, and covering spaces, J. Differential Geom. 19 (1984), 229-323.

[M1] S. Markvorsen, On the heat kernel comparison theorems for minimal submanifolds, Proc. Amer. Math. Soc. 97 (1986), 479-482.

[M2] S. Markvorsen, A characteristic eigenfunction for minimal submanifolds, Math. Z. 202 (1989), 375-382.

[M3] S. Markvorsen, On the mean exit time from a minimal submanifold, J. Diff. Geom. 29 (1989), 1-8 .

[MM] S. Markvorsen and M. Min-Oo, Global Riemannian Geometry: Curvature and Topology, Advanced Courses in Mathematics, CRM Barcelona. Birkhäuser (2003).

[MMT] S. Markvorsen, S. McGuinness, and C. Thomassen, Transient random walks on graphs and metric spaces with applications to hyperbolic surfaces, Proc. London Math. Soc. 64 (1992), 1-20 .

[MP1] S. Markvorsen and V. Palmer, Generalized isoperimetric inequalities for extrinsic balls in minimal submanifolds, J. Reine Angew. Math. 551 (2002), 101-121.

[MP2] S. Markvorsen and V. Palmer, On the isoperimetric rigidity of extrinsic minimal balls, J. Diff. Geom. Appl. 18 (2003), 47-54.

[MP3] S. Markvorsen and V. Palmer, Transience and capacity of minimal submanifolds, Geom. Funct. Anal. 13, 4 (2003), 915-933.

[MT] B. Mohar and C. Thomassen, Graphs on Surfaces, The Johns Hopkins University Press (2001).

[Nic] S. Nicaise, Approche spectrale des problèmes de diffusion sur les réseaux, Lecture Notes in Math. 1235 (1989), 120-140.

[OU] Y. Ohno and H. Urakawa, On the first eigenvalue of the combinatorial Laplacian for a graph, Interdiscip. Inform. Sci. 1 (1994), 33-46.

$[\mathrm{P}] \quad$ V. Palmer, Isoperimetric Inequalities for extrinsic balls in minimal submanifolds and their applications, J. London Math. Soc. 60, (2) (1999), 607-616.

[RS] M. Reed and B. Simon, Methods of Modern Mathematical Physics. I. Functional Analysis, Academic Press, New York (1980).

[Ro] J. P. Roth, Le spectra du Laplacien sur un graphe, Lecture Notes in Math. 1096 (1984), 521-538.

[Sa] T. Sakai, Riemannian Geometry, Translations of Mathematical Monographs, 149, American Mathematical Society, (1996). 
[So] P. M. Soardi, Potential Theory on Infinite Networks, Lecture Notes in Math. 1590, Springer-Verlag (1999).

[SmSo] U. Smilansky and M. Solomyak, The quantum graph as a limit of a network of physical wires, In 'Quantum graphs and their applications'. Contemp. Math. (Amer. Math. Soc.) 415 (2006), 283-291.

[Th] C. Thomassen, Isoperimetric inequalities and transient random walks on graphs, Ann. Probab. 20 (1992), 1592-1600.

[Ts] T. Tsuruta, Harmonic morphisms on weighted graphs, J. Math. Sci. Univ. Tokyo 7 (2000) 297-310.

[U1] H. Urakawa, A discrete analogue of the harmonic morphism, In 'Harmonic morphisms, harmonic maps, and related topics' (eds.: C. K. Anand, P. Baird, E. Loubeau, and J. C. Wood), Research Notes in Mathematics Vol. 413, Chapman \& Hall (1999), 97-108.

[U2] H. Urakawa, The Dirichlet eigenvalue problem, the finite element method and graph theory, Contemporary Mathematics, 348 (2004), 221-232.

[We] D. B. West, Introduction to Graph theory, Prentice Hall (1996).

[Wo] W. Woess, Random walks on infinite graphs and groups, Cambridge Tracts in Mathematics 138, Cambridge University Press (2000).

Department of Mathematics, Technical University of Denmark.

E-mail address: S.Markvorsen@mat.dtu.dk 